\title{
Fluorescence imaging of Evans blue extravasation into mouse brain induced by low frequency ultrasound with microbubble
}

\author{
Yuanyuan Shen $^{\mathrm{a}, \mathrm{b}, \mathrm{c}}$, Aili Zhang ${ }^{\mathrm{d}}$, Jinxuan Guo ${ }^{\mathrm{a}}$, Guo Dan ${ }^{\mathrm{a}, \mathrm{b}, \mathrm{c}}$, Siping Chen ${ }^{\mathrm{a}, \mathrm{b}, \mathrm{c}}$ and Hao Yu ${ }^{\mathrm{e}, *}$ \\ ${ }^{a}$ Department of Biomedical Engineering, Shenzhen University, Shenzhen 518060, China \\ ${ }^{\mathrm{b}}$ Guangdong Key Laboratory for Biomedical Measurements and Ultrasound Imaging, Shenzhen \\ 518060, China \\ ${ }^{\mathrm{c}}$ National-Regional Key Technology Engineering Laboratory for Medical Ultrasound, Shenzhen \\ 518060, China \\ ${ }^{\mathrm{d}}$ School of Biomedical Engineering, Med-X Research Institute, Shanghai Jiao Tong University, \\ Shanghai 200240 , China \\ ${ }^{\mathrm{e}}$ Department of Biomedical Engineering, Shenzhen Polytechnic, Shenzhen 518055, China
}

\begin{abstract}
Blood-Brain Barrier (BBB) can be opened locally, noninvasively and reversibly by low frequency focused ultrasound (FUS) in the presence of microbubbles. In this study, Evans blue (EB) dye extravasation across BBB was enhanced by $1 \mathrm{MHz}$ FUS at acoustic pressure of $0.35 \mathrm{MPa}$ in the presence of microbubbles at clinically comparable dosage. The spatial distribution of $\mathrm{EB}$ extravasation was visualized using fluorescence imaging method. The center region of BBB disruption area showed more enhanced fluorescence signal than the surrounding region in general. However, EB dye deposition was heterogeneous in the center region. The findings in this study indicated potential use of fluorescence imaging to evaluate large molecules delivery across BBB.
\end{abstract}

Keywords: Fluorescence imaging, Evans blue, Blood-Brain Barrier disruption, Focused Ultrasound, microbubble

\section{Introduction}

The existence of Blood-Brain Barrier (BBB) prevents entry of large molecules into the brain parenchyma. It is generally supposed that the permeation of molecules across BBB in significant amounts occurs unless the molecular weight is less than $400 \mathrm{Da}$ [1]. Thus, it has always been a great challenge to deliver neurotherapeutic drugs to brains for central nervous system disorders in the last decades. However, animal studies have demonstrated that BBB endothelial lining or tight junctions were opened locally, noninvasively and reversibly by Focused Ultrasound (FUS) in the presence of microbubbles in recent years [2,3].

\footnotetext{
${ }^{*}$ Corresponding author: Hao Yu, Department of Biomedical Engineering, Shenzhen Polytechnic, Shenzhen 518055, China. Tel.: +86 075526731493; Fax: +86 075526731493; E-mail: yuhao_zju@163.com.
}

0959-2989/14/\$27.50 @ 2014 - IOS Press and the authors. 
Evans blue (EB) dye is extensively used to assess the BBB permeability induced by various BBB disruption methods. EB dye has binding affinity to serum albumin. In the normal condition, the serum albumin cannot cross the BBB. However, when BBB was opened, EB-tagged albumin extravasated from vasculature into the brain tissue. Consequently, the accumulation of EB dye could be quantified after extraction from the brain tissue using spectrophotometry. However, the distribution of macromolecules passing through BBB might be heterogeneous. It was observed that there were different leakage types of Texas Red across BBB that was disrupted by ultrasound with microbubbles using two-photon fluorescence microscopy in the animal study by Cho et al. [4]. The EB dye quantification method could not assess the spatial extravasation pattern of permeability change of BBB. In recent years, fluorescence imaging technique has attracted more and more attention of researchers to study animal brain vasculature leakage in stroke or cerebral ischemia models $[5,6]$. The results showed that global ischemic damage pattern could be visualized using fluorescence imaging method, which was much more sensitive than conventional ultraviolet spectrophotometry [5].

In the present study, the blood-brain barrier was disrupted noninvasively by low frequency FUS in the presence of microbubbles. The feasibility of visualizing EB-binding serum albumin extravasation from BBB was investigated using fluorescence imaging method. The fluorescence images of the spatial distribution of EB dye were compared with light photographs and expected to provide basic information for strategies of delivering large molecules to brains.

\section{Materials and methods}

\subsection{Animal preparation}

Six-week-old female Balb/c mice (20 $\pm 2 \mathrm{~g}$ ) were bought from Guangdong Medical Laboratory Animal Center, Foshan, China. They were housed in sterile isolated cages with a $12 \mathrm{~h}$ light/dark cycle at constant temperatures $\left(24-26^{\circ} \mathrm{C}\right)$ and humidity $(30-50 \%)$. Sterilized food and water were provided. Animal care and experiments were performed in accordance with the guidelines for the Animal Care and Use Committee of School of Medicine in Shenzhen University (SZU-HC-2014-01).

Prior to sonication, each mouse hair was removed clearly with depilatory cream. Then they were anesthetized with $1 \%$ pentobarbital sodium $(5 \mu \mathrm{L} / \mathrm{g}$ of body weight) intraperitoneally, and placed on a heating pad with a constant temperature $37^{\circ} \mathrm{C}$ to maintain their body temperature. Four mice were sonicated transcranially using FUS with microbubbles. One mouse was sonicated using FUS only and one mouse underwent the whole procedure without FUS.

\subsection{Microbubble generation}

Lipid-shelled microbubbles with perfluoropropane gas core were prepared as described by Tinkov [7]. The shell was coated with DPPC: DPPG: DSPE-PEG2000 (Avanti Polar Lipids) at a molar ratio of 76:19:5. The phospholipids were dissolved in chloroform (Sinopharm Chemical Reagent Col, Ltd) and the organic solvent was removed under vacuum. And 0.01 M phosphate buffer saline (PBS) solution was mixed with $10 \mathrm{vol} \%$ glycerol solution and $10 \mathrm{vol} \%$ propylene glycol (Sinopharm Chemical Reagent Col, Ltd). Then the dried phospholipid film was hydrated with PBS mixture to a final total phospholipid concentration of $5 \mathrm{mM}$. The lipid suspension was heated to $60^{\circ} \mathrm{C}$ and sonicated in a water bath to disperse the lipid aggregates. After sealing within 3-mL glass serum vial, the air headspace was exchanged with perfluoropropane gas (99.999\% purity, Suzhou Xundong gas technology Co., Ltd). 
The microbubbles were generated by agitating the vial mechanically at the speed of 4500 oscillations/min for 20 seconds.

\subsection{Sonication procedure}

FUS wave was generated by a single-element spherical transducer made in lab (Center frequency: $1.051 \mathrm{MHz}$, focal length: $90 \mathrm{~mm}$, diameter: $38 \mathrm{~mm}$ ). The FUS transducer was driven by a power amplifier (A150, Electronics \& Innovation, Rochester, NY, USA) and mounted at the bottom of an acrylic water tank filled with degassed water.

The pressure amplitude was measured using a membrane hydrophone (HMB-0500, Onda Corporation) in degassed water tank. The peak-rarefactional pressure amplitude without correction due to skull attenuation was $0.43 \mathrm{MPa}$ in this study. The full width at half maximum in the lateral diameter of the beam was $4.75 \mathrm{~mm}$. Pulsed FUS was applied with a burst length of $10 \mathrm{~ms}$ and a repetition frequency of $1 \mathrm{~Hz}$. The duration of the sonication was $60 \mathrm{~s}$.

Before sonication, the mice were placed in the supine position on a sonication table above the water tank. A $0.1 \mathrm{~mL}$ bolus of diluted microbubble solution was injected through tail vein at a dose of 25 $\mu \mathrm{L} / \mathrm{kg}$ of body weight and circulated for about $15 \mathrm{~s}$. Then EB $(2 \%, 5 \mathrm{~mL} / \mathrm{kg})$ was injected through the tail vein of each mouse for about 1 min after sonication. Two hours after EB injection, all the mice were anesthetized and perfused with saline through the left ventricle until clear perfusion fluid flooded from the right atrium. Then $4 \%$ freshly prepared paraformaldehyde solution (PFA) was perfused. After heart perfusion, the brain tissues were extracted from the skull for fluorescence imaging.

\subsection{Fluorescence imaging method}

The fluorescence of EB was visualized by an optical imaging system IVIS Lumina II (PerkinElmer). The extracted brain tissues were placed in the scanning chamber of the imaging system. Spectral unmixing mode was used with excitation filters that span 535-640 nm and emission filter Cy5.5.

\subsection{Histology}

The excised brain tissues were fixed in $10 \%$ formalin solution for at least $24 \mathrm{~h}$. After washing and dehydrating, they were embedded in paraffin and sliced to a thickness of $7 \mu \mathrm{m}$. Hematoxylin-eosin $(\mathrm{H}$ $\&$ E) and Cresyl violet (Nissl staining) stains are commonly used histological methods for the morphological assessments of the cells in brain tissue. Thus the paraffin slices were stained with $\mathrm{H} \& \mathrm{E}$ and and Cresyl violet in the study for histologic evaluation.

\section{Results}

\subsection{Microbubble size distribution}

Microbubble size distribution and concentration were determined by Multisizer 4 Coulter Counter (Beckman Coulter). The aperture diameter was $30 \mu \mathrm{m}$ and the sampling solution was diluted with Iston II. The size distribution and concentration of microbubble samples were determined immediately and seven days after preparation respectively. Figure 1 shows the size distribution of prepared microbubbles. The diameters of most microbubbles were less than $8 \mu \mathrm{m}$. 


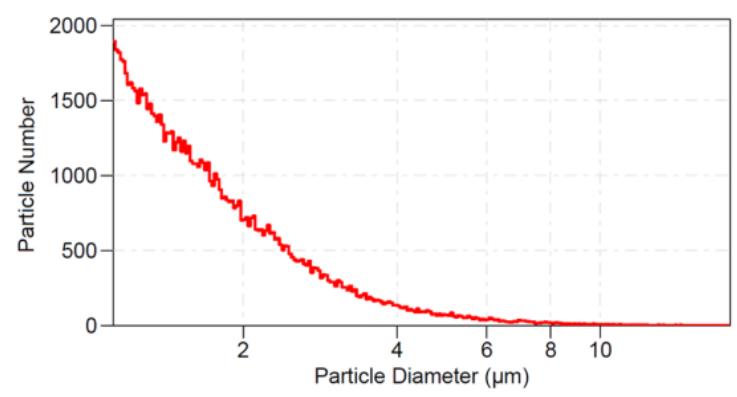

Fig. 1. The size distribution of prepared microbubbles.

Table 1

The measurement data of prepared microbubbles

\begin{tabular}{|l|l|l|l|}
\hline Microbubble samples & Mean diameter & Concentration & $\mathrm{d} 90 *$ \\
\hline Freshly prepared & $1.92 \mu \mathrm{m}$ & $4.101 \times 10^{9} / \mathrm{mL}$ & $2.98 \mu \mathrm{m}$ \\
\hline Seven days after preparation & $1.99 \mu \mathrm{m}$ & $1.331 \times 10^{9} / \mathrm{mL}$ & $3.17 \mu \mathrm{m}$ \\
\hline
\end{tabular}

Note: $\mathrm{d} 90^{*}$ means that the amount of the particles with diameters less than $2.98 \mu \mathrm{m}$ takes up $90 \%$ of the total number.

Table 1 summarized measurement data. The mean diameter of freshly prepared microbubble was $1.92 \mu \mathrm{m}$, and increased to $1.99 \mu \mathrm{m}$ seven days after preparation. The amount of the microbubbles with diameters less than $2.98 \mu \mathrm{m}$ accounted for $90 \%$ of the total number as indicated by the parameter $\mathrm{d} 90$ in Table 1, and the diameter increased to $3.17 \mu \mathrm{m}$ seven days after preparation. The measurement results suggested that the prepared microbubbles be stable. The concentration was $4.101 \times 10^{9} / \mathrm{mL}$ and decreased to $1.331 \times 10^{9} / \mathrm{mL}$ after 7 days. Compared with Sonovue microbubbles $\left(5.0-8.0 \times 10^{8} / \mathrm{mL}\right)$, it was dense enough for animal study.

\subsection{EB dye extravasation due to BBB disruption}

The photographs of the mouse brain harvested two hours after EB injection were shown in Figure 2. Slight blue coloration spot of the brain due to EB extravasation was visible, indicating that BBB was successfully opened by low frequency FUS with microbubbles. In the study, four mice were treated with low frequency FUS in the presence of microbubbles. The successful rate of BBB opening was $100 \%$.

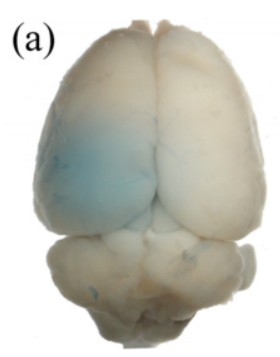

(b)

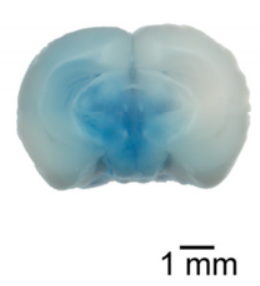

Fig. 2. Photographs of EB dye extravasation due to BBB disruption by low frequency FUS with microbubbles. (a) top surface; (b) coronal slice. Bar: $1 \mathrm{~mm}$. 
Fluorescence images of EB dye extravasation of four mouse brains as shown in Figure 3(b) were compared with the photograph images as shown in Figure 3(a). In both Figures, the upper left mouse brain was treated without FUS and microbubbles as the control; the upper right mouse brain was treated with FUS only; while the lower left and right mouse brains were treated with FUS in the presence of microbubbles at the same sonication protocol. For the two upper mouse brain tissues, blue coloration spots and fluorescence signals were not seen, indicating there was no BBB disruption. While in the presence of microbubbles, blue coloration spots and fluorescence signals were clearly visible for the two lower mouse brains, revealing that BBB was opened by low frequency FUS with microbubbles. Fluorescence image showed spatial distribution of EB dye extravasation. In the center region of the BBB disrupted area, the fluorescence signal of extravasated EB was more significant than that in the peripheral region.

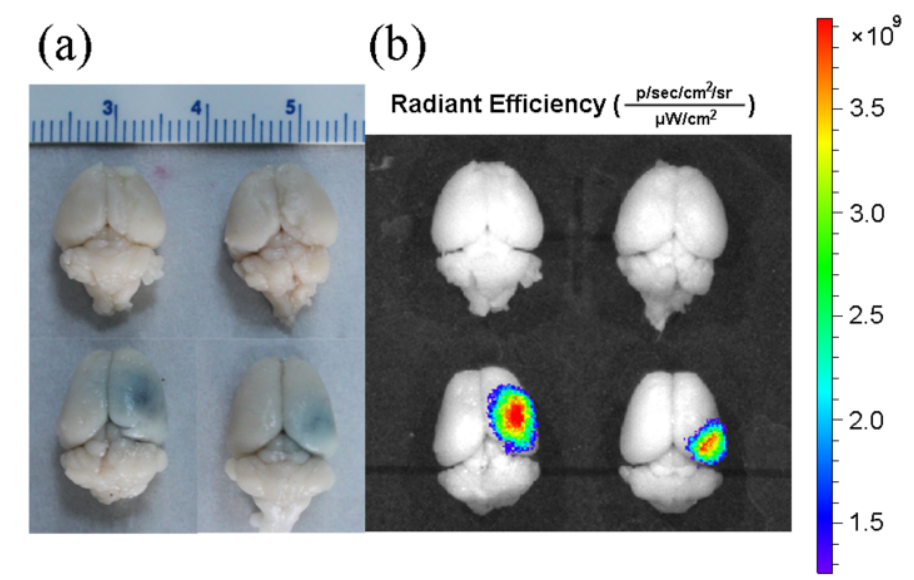

Fig. 3. Images of EB dye extravasation due to BBB disruption by low frequency FUS with microbubbles. The upper left mouse brain was treated without FUS and microbubbles as the control. The upper right mouse brain was treated with FUS only. The lower left and right mouse brains were treated with FUS in the presence of microbubbles at the same sonication protocol. (a) photograph image; (b) fluorescence image.

(a)

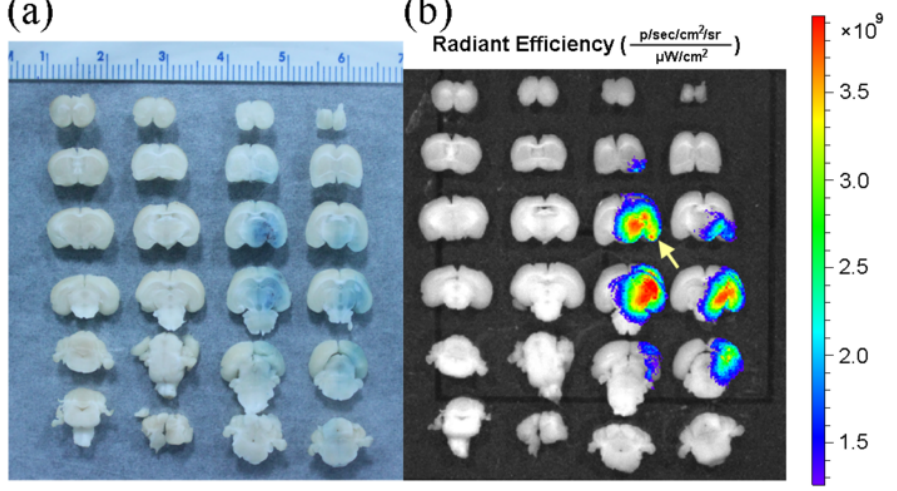

Fig. 4. Images of brain coronal slices of EB dye extravasation due to BBB disruption by low frequency FUS with microbubbles. The first left column was the control mouse. The second left column was the mouse treated with FUS only. The two right columns were the mice treated with FUS and microbubbles. The yellow arrow indicated heterogeneous distribution of extravasated EB in BBB disruption area. (a) photograph image; (b) fluorescence image. 

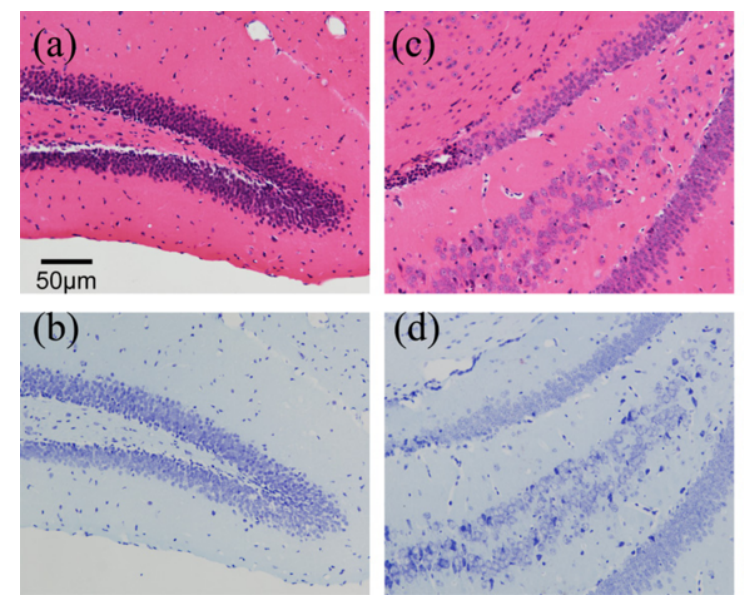
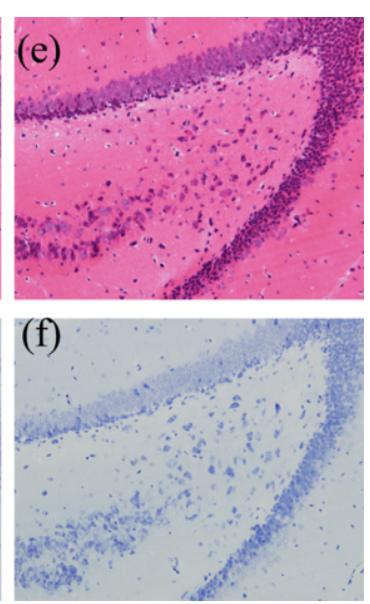

Fig. 5. Histology examinations of brain tissues of mice sonicated transcranially. (a-b) control; (c-d) using FUS only; (e-f) using FUS with microbubbles. The upper row: H \& E staining; The lower row: Nissl staining. Bar: $50 \mu \mathrm{m}$.

After imaging the top surface of the intact brains, all mouse brain tissues were sectioned into six slices and mounted with coronal planes horizontally in a glass dish. Figures 4(a) and 4(b) illustrated both photograph and fluorescence images of all slices, respectively. In both images, the first left column was the control mouse; the second left column was the mouse treated with FUS only, and the two right columns were the mice treated with FUS and microbubbles. In the fluorescence image as shown in Figure 4(b), most brain slices showed a central enhancement pattern of EB deposition extravasated from brain vasculature. However, the third slice of the third column from left to right showed heterogeneous distribution of extravasated EB in BBB disrupted area as indicated by the yellow arrow.

\subsection{Histology}

In the present study, both H\&E staining and Nissl staining were used to evaluate the cellular structure of the brain tissue. Figure 5 showed histology of the mouse brain tissues sonicated transcranially. There was no hemorrhage and red blood cell extravasation as illustrated in Figure 5(e). The image of Nissl staining showed mottled purple-blue Nissl body in the cytoplasm, demonstrating that no acute neuron damage occurred as seen in Figure 5(f). The histology examinations indicated that the sonication parameters used in the present study were safe.

\section{Discussion and conclusion}

In the recent decade, experimental studies have suggested that low frequency FUS with microbubbles is a promising potential method to open BBB noninvasively, locally and reversibly. There are many critical parameters correlated with BBB opening, such as microbubble concentration, acoustic pressure, burst length, pulse repetition frequency, etc. In the present study, the peak-rarefactional pressure after derating by $18 \%$ due to mouse skull attenuation was $0.35 \mathrm{MPa}$ [2]. Microbubbles were administered systemically at a dose of $25 \mu \mathrm{L} / \mathrm{kg}$ of body weight. This dosage was comparable to the clinical dose (10 $\mu \mathrm{L} / \mathrm{kg}$ of body weight). The molecular weight of murine serum albumin was $67 \mathrm{kDa}$. EB dye extravasation into brain tissue indicated that the serum albumin penetrated BBB. In the most re- 
cent study by Chen et al., fluorescently labelled dextran with molecular weight of $70 \mathrm{kDa}$ was delivered successfully across BBB of four mice at $0.51 \mathrm{MPa}$, which was larger than the acoustic pressure used in our study [8]. In their study, the microbubbles were used at a dose of $50 \mu \mathrm{L} / \mathrm{kg}$ of body weight, which was two-fold of the dosage in our study. Thus, the acoustic parameters and microbubble dosage should be investigated in further study.

Contrast-enhanced magnetic resonance imaging (MRI) with gadodiamide has been employed to monitor the BBB opening in vivo in many other studies [3]. However, gadodiamide has no binding affinity to plasma albumin and its molecular weight ( $\sim 550 \mathrm{Da})$ is much smaller than serum albumin $(67 \mathrm{kDa})$. Differences in the spatial extravasation pattern of gadodiamide and EB have been reported previously [9]. Due to its low molecular weight, contrast-enhanced MRI with gadodiamide might not be a suitable method to assess the BBB opening for large molecule leakage. Since many therapeutic agents could be fluorescently labeled, the results of this study demonstrated the feasibility of assessing BBB disruption using fluorescence imaging.

Despite a number of studies in BBB disruption induced by FUS with microbubbles, investigations on the spatial distribution of the agents across BBB are limited. In the present study, EB dye distribution extravasated from brain vasculature was heterogeneous in the center region. In a study by Choi et al., it was found that greater contrast enhancement in the vicinity of arteries was detected than that in homogeneous brain tissue using contrasted MRI imaging [10]. Thus, the correlation of brainvasculature with the spatial distribution of large molecules should be explored for effective and safe drug delivery to central nervous system disorders.

In the present study, the BBB was disrupted noninvasively and locally by $1 \mathrm{MHz}$ FUS at the acoustic pressure of $0.35 \mathrm{MPa}$ in the presence of microbubbles at clinically comparable dosage. The EBbinding serum albumin extravasation from BBB was visualized using fluorescence imaging method. The center region of BBB disruption area showed more enhanced fluorescence signal than the surrounding region in general. However, EB dye distribution extravasated from brain vasculature was heterogeneous in the center region. The findings in this study indicated potential use of fluorescence imaging to evaluate large molecule delivery across BBB.

\section{Acknowledgement}

This work is supported by grants from National Natural Science Foundation of China (Grant No. 61372006, 61001057) and Shenzhen Science and Technology Planning Project (Grant No. GJHS20120621153609166). The authors would like to thank Shashank Sirsi (Department of Chemical Engineering, Columbia University, USA) and Jian Chen (Department of Pharmacy, Shanghai Jiao Tong University, China) for the suggestions and assistance in the microbubble preparation.

\section{References}

[1] W.M. Pardridge, The blood-brain barrier: bottleneck in brain drug development, NeuroRx. 2 (2005), 3-14.

[2] J.J. Choi et al., Noninvasive transcranial and localized opening of the blood-brain barrier using focused ultrasound in mice, Ultrasound Med. Biol. 33 (2007), 95-104.

[3] K. Hynynen et al., Noninvasive MR imaging-guided focal opening of the blood-brain barrier in rabbits, Radiology 220 (2001), 640-646.

[4] E.E. Cho et al., Two-photon fluorescence microscopy study of cerebrovascular dynamics in ultrasound-induced bloodbrain barrier opening, J. Cereb. Blood Flow Metab. 31 (2011), 1852-1862.

[5] H. Jaffer, I.M. Adjei and V. Labhasetwar, Optical imaging to map blood-brain barrier leakage, Sci. Rep. 3 (2013), 3117. 
[6] J. Klohs et al., Near-infrared fluorescence imaging with fluorescently labeled albumin: a novel method for non-invasive optical imaging of blood-brain barrier impairment after focal cerebral ischemia in mice, J. Neurosci. Methods 180 (2009), 126-132.

[7] S. Tinkov, Development of ultrasound contrast agents for targeted drug and gene delivery, Ph.D. Dissertation, LudwigMaximilians-University Munich, 2009.

[8] H. Chen and E.E. Konofagou, The size of blood-brain barrier opening induced by focused ultrasound is dictated by the acoustic pressure, J. Cereb. Blood Flow Metab. 34 (2014), 1197-1204.

[9] T.N. Nagaraja et al., Identification of variations in blood-brain barrier opening after cerebral ischemia by dual contrastenhanced magnetic resonance imaging and T 1sat measurements, Stroke 39 (2008), 427-432.

[10] J.J. Choi et al., Spatio-temporal analysis of molecular delivery through the blood-brain barrier using focused ultrasound, Phys. Med. Biol. 52 (2007), 5509-5530. 